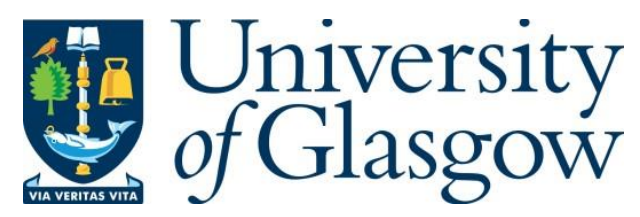

Liang, J., Lee, J., Berrada, S., Georgiev, V., Asenov, A., Azemard-Crestani, A. and Todri-Sanial, A. (2018) Atomistic to Circuit Level Modeling of Defective Doped SWCNTs with Contacts for On-Chip Interconnect Application. In: 12th IEEE Nanotechnology Materials and Devices Conference (NMDC 2017), Singapore, 2-4 Oct 2017, pp. 66-67. ISBN 9781538627723.

There may be differences between this version and the published version. You are advised to consult the publisher's version if you wish to cite from it.

http://eprints.gla.ac.uk/151261/

Deposited on: 30 November 2018

Enlighten - Research publications by members of the University of Glasgow http://eprints.gla.ac.uk 


\title{
Atomistic to Circuit Level Modeling of Defective Doped SWCNTs with Contacts for On-Chip Interconnect Application
}

\author{
J. Liang ${ }^{1}$, J. Lee ${ }^{2}$, S. Berrada ${ }^{2}$, V. Georgiev ${ }^{2}$, A. Asenov ${ }^{2}$, N. Azemard-Crestani ${ }^{1}$, A. Todri-Sanial ${ }^{1}$ \\ CNRS-LIRMM/University of Montpellier, France ${ }^{1}$ \\ School of Engineering, University of Glasgow, $\mathrm{UK}^{2}$
}

\section{INTRODUCTION}

Carbon nanotubes (CNTs) due to their high electrical/thermal conductivity, high ampacity, high tolerance to electromigration [1] and small dimensions make them an ideal candidate for future on-chip interconnects [2]. Fabricating the CNTs, random chirality and some defects are introduced which can degrade the CNT electrical properties [3]. Additionally, the contact resistance between metal and CNT presents additional parasitics that impose restraints on the electron transport. Electrical models of CNT for interconnect application were developed several years ago [4-5]. In this paper, we explored on doped and defective single-wall CNTs (SWCNT $(24,0)$ ) including contact resistance as important physical parameters to assess the performance of fabricated SWCNTs realistically for back-end-of-line (BEOL) on-chip interconnects on VLSI circuit application.

\section{HIERARCHAL MODEL}

Fig. 1 shows the atomistic structure of SWCNT $(24,0)$. In order to extract the defective resistance with this structure, we have performed tight-binding (TB) - Non-Equilibrium Green's Function (NEGF) simulations implemented in the Atomistix ToolKit (ATK) [6-7]. The length of central region is set to about 42.6nm having 9600 carbon atoms. We distributed vacancy defects in central region randomly from 1 to 6 , and have calculated the defective resistance by $R_{\text {defect }}=R-$ $R_{\text {perfect }}$. The dependence of $R_{\text {defect }}$ on the number of defects is shown in Fig. 2. For statistical analysis, 80 samples were used. We have found that the median value of $R_{\text {defect }}$ varies from 4 to $40 \mathrm{k} \Omega$ with 1 to 6 defects, respectively.

We have calculated the number of conducting channels $N_{C}$ of doped SWCNT $(24,0)$ using Density Functional theory (DFT) with generalized gradient approximation (GGA). It was assumed that the dopant only changed the Fermi level of the CNT without affecting its band structure. The number of conducting channel is derived by $G / G_{0}$, where $G_{0}$ is the quantum conductance $2 e^{2} / h\left(G_{0}=7.748 \cdot 10^{-5} \mathrm{~S}\right)$. Thanks to the DFT-GGA calculation, we have found that $N_{C}$ can vary from 2 to 8 when the Fermi level is changed from -1.0 to $1.0 \mathrm{eV}(0.0$ $\mathrm{eV}$ is set to the Fermi level of pristine CNT.).

Based on previous works, SWCNT interconnect can be represented by an $R C$-model. By taking into the account the impact of doping, defects and contact resistance, we compute the SWCNT resistance as follows:
$R_{S W C N T}=\frac{h}{2 e^{2} N_{c}}\left(1+\frac{L}{L_{m f p}}\right)+R_{\text {defect }}+R_{\text {Contact }}, \quad$ where $L_{m f p}$ and $L$ are the electron mean free path and the interconnect length, respectively [8]. Moreover, the parasitic capacitance can be written by $C_{S W C N T}=\frac{C_{Q} C_{E}}{C_{Q}+C_{E}}$, where $C_{Q}$ and $C_{E}$ are quantum and electrostatic capacitances, respectively [4]. We use a circuit benchmark as shown in Fig. 3 to investigate SWCNT's performance. $R_{\text {driver }}, C_{\text {driver }}$ and $C_{\text {load }}$ are assumed to be $24 \mathrm{k} \Omega, 450 \mathrm{aF}$ and $10 \mathrm{fF}$, respectively, which are values extracted from the logic gates implemented in CMOS $45 \mathrm{~nm}$ technology node using Cadence environment with Verilog-A models.

\section{SIMULATION RESULTS}

Fig. 4 shows the delay ratio of the doped and defective SWCNT $(24,0)$ to the perfect SWCNT. SWCNT interconnect length sets to be $1 \mu \mathrm{m}$. As shown in the figure, the propagation delay $\left(t_{d}\right)$ decreases as $N_{C}$ increases by dopants. This is because the interconnect resistance decreases due to larger $N_{C}$. In this analysis, the change of $C_{Q}$ due to the Fermi level shift had no significant effect. However, additional defective resistance increases the propagation delay. We observed that $t_{d}$ increases by about $6 \%$ due to 6 defects, and increasing $N_{C}$ to 8 reduces it to $5 \%$. Moreover, the delay ratio of doped SWCNT $\left(N_{C}=3\right)$ with 1 defect decreases to 1 , which means that it has the same $t_{d}$ as the perfect SWCNT. Such results indicate that doped CNTs can mitigate the increase in $t_{d}$ caused by defects. However, if there are two or more defects, it is not possible to return the delay ratio to 1.0 even with high doping concentration. Thus, it is more important to not create too much defects during the fabrication process.

Fig. 5 (a) describes the dependence of the delay ratio of the doped SWCNT on the contact resistance when $L=1 \mu \mathrm{m}$. In order to keep the delay ratio less than 1 , the contact resistance should be less than $4 \mathrm{k} \Omega(8 \mathrm{k} \Omega)$, and $N_{C}$ should be greater than 3 (8). However, if $L=10 \mu \mathrm{m}$, the delay ratio is less than 1 even though the contact resistance and $N_{C}$ are $10 \mathrm{k} \Omega$ and 3 , respectively as shown in Fig. 5 (b). Given the time delay dependence on $L$, we found that doping process has a greater impact to enhance the conductance when the interconnect length is longer. Therefore, for the efficient circuit, one should focus on reducing the contact resistance as the interconnect length is shorter. 


\section{CONCLUSION}

A hierarchical model, from atomistic to electrical compact model is presented and investigated. We calculate the defective resistance and number of conducting channels of doped SWCNT using TB and DFT-GGA approaches. Through the circuit-level electrical compact modeling and simulations, we investigate the impact of defects and doping on the SWCNT interconnect in terms of the propagation delay. We believe that our study provides the guidelines for the optimal design of CNT interconnects.

\section{ACKNOWLEDGMENT}

Authors would like to thank H2020 CONNECT European project. This project has received funding from the European Union's Horizon 2020 research and innovation program under grant agreement No 688612. (http://www.connect-h2020.eu/)

\section{REFERENCES}

[1] C. Subramaniam et al. Nat.Commun. 4, 2202 (2013).

[2] A. Todri-Sanial et al., Carbon Nanotube Interconnects: Process, Design and Applications, Springer 2016, ISBN\#: 978-3-319-29744-6.

[3] M. Bockrath et al. Science, 291, 283-285 (2001).

[4] Srivastava et al. ICCAD-2005. IEEE/ACM (2005).

[5] L. Hong et al. IEEE Transactions on electron devices55.6 (2008) : 1328-1337.

[6] Atomistix Tool Kit (2016.3), QuantumWise A/S.

[7] Y. Hancock et al. J Low Temp Phys, 153, 393-398 (2008).

[8] J. Jiang et al. Physical Review Letter, vol. 64, no. 4, 2 July 2001.

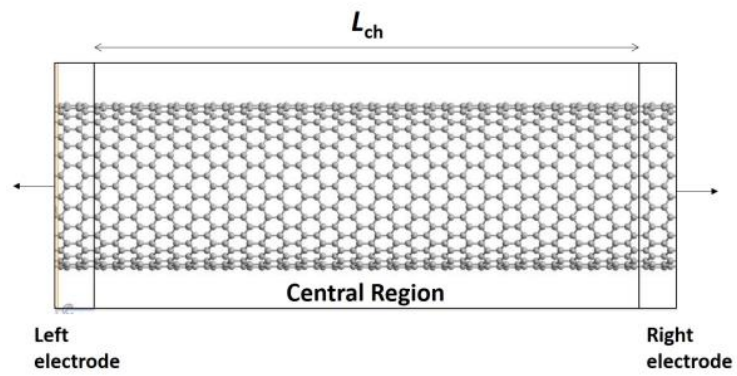

Figure 1. Atomistic structure of SWCNT $(24,0)$ interconnect.

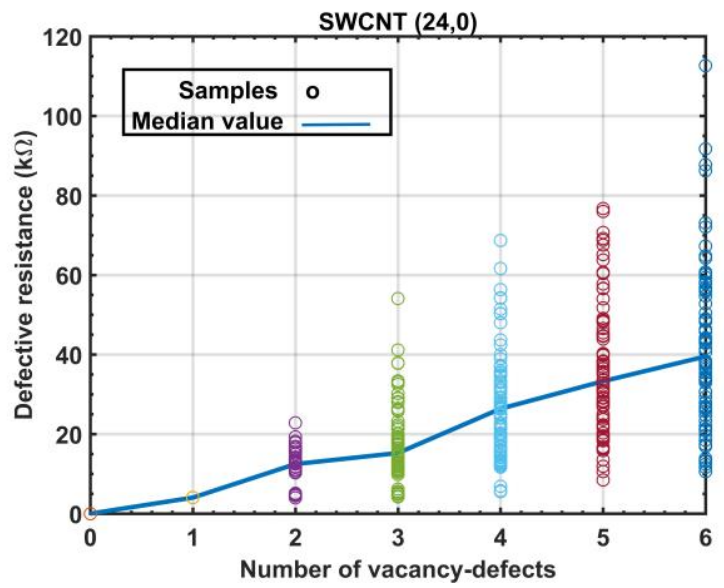

Figure 2. Dependence of defective resistance on number of vacancy defects for SWCNTs $(24,0)$. We used 80 samples for the statistical analysis.

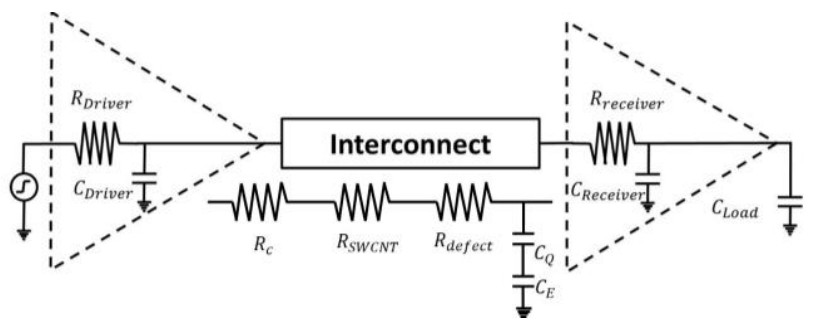

Figure 3. Schematic diagram for the circuit-level simulation.

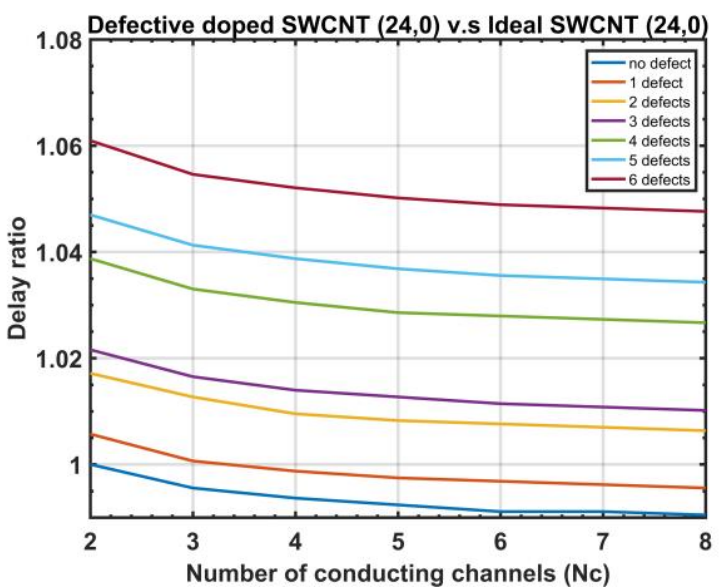

Figure 4. Dependence of the delay ratio of defective $\operatorname{SWCNT}(24,0)$ to perfect SWCNT $(24,0)$ on the number of conducting channels.

Doped SWCNT $(24,0)$ with contact resistance v.s Ideal SWCNT $(24,0)$

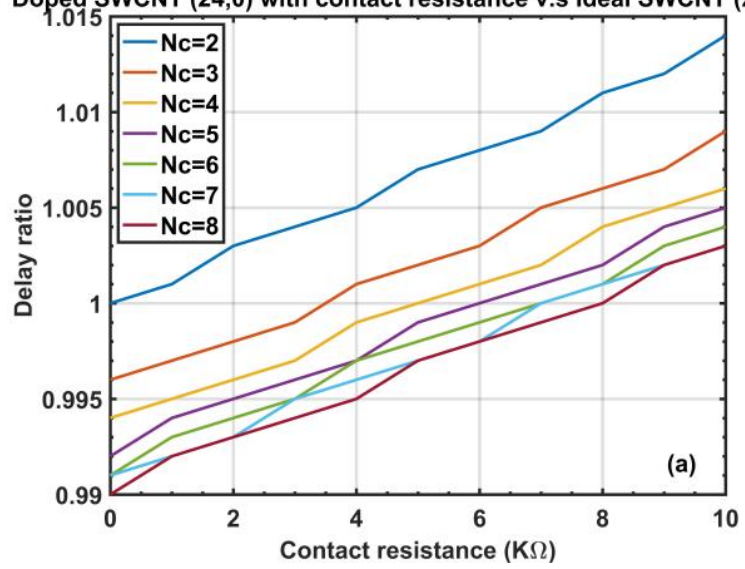

Doped SWCNT $(24,0)$ with contact resistance vs. Ideal SWCNT $(24,0)$

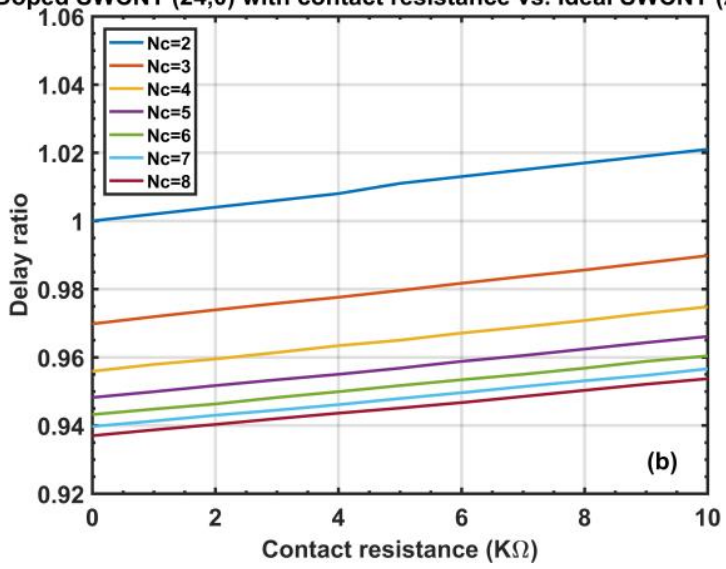

Figure 5. Dependence of the delay ratio of doped SWCNT to perfect SWCNT on the contact resistance. Interconnect lengths are (a) $1 \mu \mathrm{m}$ and (b) $10 \mu \mathrm{m}$, respectively. 